\title{
A novel procedure for protein extraction from formalin-fixed paraffin-embedded tissues
}

\author{
Teresa Rodríguez-Rigueiro ${ }^{1}$, Manuel Valladares-Ayerbes ${ }^{1,2}$, Mar Haz-Conde1, \\ Moisés Blanco ${ }^{1}$, Guadalupe Aparicio ${ }^{1}$, Patricia Fernández-Puente ${ }^{3}$, Francisco J. \\ Blanco $^{3}, \mathrm{M}^{\mathrm{a}}$ José Lorenzo $^{2}$, Luis A. Aparicio ${ }^{1,2}$ and Angélica Figueroa ${ }^{1}$
}

1. Translational Cancer Research Group, Proteo-Red-ISCIII, Instituto de Investigación Biomédica A Coruña (INIBIC), Coruña, Spain

2. Medical Oncology Unit, Complejo Hospitalario Universitario A Coruña (CHUAC), Coruña, Spain

3. Proteomics Unit, Proteo-Red-ISCIII, Instituto de Investigación Biomédica A Coruña (INIBIC), Coruña, Spain

\begin{abstract}
Most of the archived pathological specimens in hospitals are kept as formalin-fixed paraffin-embedded tissues (FFPE) for long-term preservation. Up to now, these samples are only used for immunohistochemistry in a clinical routine as it is difficult to recover intact protein from these FFPE tissues. Here, we report a novel, short time-consuming and cost-effective method to extract full-length, non-degraded proteins from FFPE tissues. This procedure is combined with an effective and non-toxic deparaffinisation process and an extraction method based on antigen-retrieval, high concentration of SDS and high temperature. We have obtained enough intact protein to be detected by Western blotting analysis. This technique will allow utilising these stored FFPE tissues in several applications for protein analysis helping to advance the translational studies in cancer and other diseases.
\end{abstract}

Keywords:

Biomedicine; Cancer; FFPE; Human samples; Protein expression; Western blotting 
In the last few years, biomedical investigations have been focussing on the improvement of the translational research which tries to integrate the findings on basic science more quickly and efficiently into medical practice. At present, the barriers between molecular biology and clinical trials are narrower, thanks to the multi-disciplinary collaboration between scientists and clinicians. For instance, the establishment of a huge number of tissue banks in hospitals for more than a century is one of the most important benefits that scientist can take for their biomedical research. Human tissues obtained from biopsies are generally fixed in formalin and embedded in paraffin (FFPE) to be further analysed by a histopathology expert. FFPE is a standard method for long-term preservation of most archived pathological specimens. Moreover, these FFPE tissue collections are also important resources to develop studies on which DNA and RNA are the starting materials 1. However, research work is still limited on the extraction of intact protein from FFPE tissues as it is believed that their processing with formalin and paraffin destroys proteins and makes their analysis difficult by Western blotting, immunoprecipitation or protein arrays. Western blotting and immunohistochemistry (IHC) are the most frequent techniques used to identify and study protein expression. Although IHC gives information regarding the localisation and distribution of proteins in cells and tissues, it is not adequate for protein quantification as there is an important risk of cross-reacting proteins.

In 1998, Ikeda et al. 2 published the first work on which they obtained intact protein from FFPE tissues; however, they failed to obtain comparable extracts from FFPE and fresh-frozen tissues. Since then, not many works have been published describing approaches to obtain fulllength protein for expression analysis 3-6. It is well known that not all the proteins can be extracted under the same conditions. The extraction of individual proteins can be proceeded in several manners considering that the solubilisation of some proteins require conditions that destabilise others. In this article, we describe a novel standardised and reproducible method for full-length protein extraction from FFPE tissues. By Western blotting, we have shown that we obtain comparable yields to that seen from fresh-frozen tissue samples. This method can be used to compare protein expression from different tissues, individuals and pathological conditions.

Human tissues obtained from biopsies were collected under informed consent from all patients; this work was approved by the local research ethics committee. We used not matched $-80^{\circ} \mathrm{C}$ frozen and FFPE blocks, collected from the research tissues tumour bank of our institute (INIBICCHUAC) and from the pathological anatomy department from the hospital (CHUAC), respectively. All the samples used were stored for $<5$ years to reduce variations by long-term storage. For each extraction, six series of $15 \mu \mathrm{m}$ sections from frozen and FFPE, with an area of $\sim 150 \mathrm{~mm}^{2}$ each, were collected in an Eppendorf tube. The frozen samples were pulverised in liquid nitrogen and the FFPE were deparaffinised using mineral oil. Several investigators have successfully extracted intact protein from FFPE samples stored for years by using the conventional xylene/ethanol procedure for deparaffinisation 5, 7; however, it is more time consuming and more toxic protocol, and the deparaffinisation procedure is not always complete. Six hundred microlitres of mineral oil was added (Sigma-Aldrich, USA); after vortexing, samples were incubated at $95^{\circ} \mathrm{C}$ for $2 \mathrm{~min}$ to dissolve the wax, and finally supernatants were removed after pelleting at $14000 \times g$ for $3 \mathrm{~min}$ at room temperature (RT). These incubation and centrifugation steps were repeated again by adding $350 \mu \mathrm{L}$ of mineral oil. The pellets were resuspended in $200 \mu \mathrm{L}$ of the indicated buffers (see below) to wash the samples, and after centrifugation at $14000 \times \mathrm{g}$ for $3 \mathrm{~min}$, they were incubated in $50 \mu \mathrm{L}$ of the specific buffer to continue with the protein extraction procedure. The deparaffinisation protocol using mineral oil was recently described for the high quality of DNA extraction suitable for genotyping experiments 8. Two different extraction buffers were first used: urea-lysis buffer (ULB: $30 \mathrm{mM}$ Tris-base, $8 \mathrm{M}$ urea, $2 \mathrm{M}$ thiourea and 4\% CHAPS) and SDS-lysis buffer (SDS-LB: $200 \mathrm{mM}$ Tris-HCl, pH 6.8, 20\% glycerol and 2\% SDS). Samples were incubated with these buffers for $1 \mathrm{~h}$ at RT with shaking. After centrifugation at $14000 \times \mathrm{g}$ for $15 \mathrm{~min}$ at RT, supernatants were recovered. Protein quantification for ULB was carried out by Bradford method 9 with the EZQ Protein Quantitation Kit (Molecular Probes, Eugene, OR, USA) and for SDS-LB, quantification was performed by using BCA protein assay kit (Pierce, USA) according to manufacturer's instructions. Forty micrograms of extracted lysates was resolved in $10 \%$ polyacrylamide SDS-PAGE according to Laemmli 10 in a BioRad Mini Protean Tetra Cell system (Herts, UK). For Western blotting, electrophoresed proteins were transferred onto a PVDF membrane (Immobilon-P, Millipore) at $200 \mathrm{~mA}$ for $1 \mathrm{~h}$. The membranes were blocked in PBS plus 
3\% skim milk for $1 \mathrm{~h}$ at RT with constant shaking. They were incubated with the indicated antibodies at RT for $1 \mathrm{~h}$, washed three times with PBS plus $0.05 \%$ Tween-20 (PBS-T) and incubated for $1 \mathrm{~h}$ with the specific secondary anti-mouse or anti-rabbit peroxidase-conjugated antiIgG antibody. After three washes with PBS-T, the proteins were visualised for immunological detection by enhanced chemiluminescence ( $\mathrm{ECL}^{+}$; GE Healthcare, Sweden). Antibodies used in this work were as follows: mouse $\alpha$-tubulin antibody (Sigma-Aldrich), rabbit Hakai antibody kindly provided by Yasuyuki Fujita (LMCB, UK) 11, E-cadherin (Invitrogen Laboratories, CA) and HRP-rabbit and mouse polyclonal antibodies (GE Healthcare, UK). Primary and secondary antibodies were used at dilutions 1:1000 and 1:5000, respectively.

As shown in Fig. 1A, we used $\alpha$-tubulin-based normalisation to compare the RT extraction quality from fresh-frozen and FFPE colon carcinoma tissues. It is well known that the normalisation of a loading control to compare protein expression in different tissues and in different conditions such as tumour/normal profiles is crucial for optimisation and validation of reproducible results. Under the above explained extraction conditions, $\alpha$-tubulin was detected in fresh-frozen tissues using both extraction buffers; however, these buffers were not efficient to detect $\alpha$-tubulin in FFPE colon carcinoma, compared with that seen in fresh-frozen tissues. Therefore, we conclude that these RT extraction methods are not effective for the correct protein extraction from FFPE tissues. It was previously described that high concentration of SDS and high temperature are important conditions required to obtain an efficient protein extraction from FFPE tissues 2. Taking into consideration these premises and the importance of the antigen retrieval by sodium citrate used in IHC, we implemented a novel extraction buffer and protocol that can obtain similar intact protein in FFPE and in fresh-frozen tissues. Fifty microlitres of buffer, containing $200 \mathrm{mM}$ Tris- $\mathrm{HCl}, \mathrm{pH} 7.5,200 \mathrm{mM} \mathrm{NaCl}, 5 \%$ SDS and $100 \mathrm{mM}$ sodium citrate, was used to incubate the samples at $100^{\circ} \mathrm{C}$ for $20 \mathrm{~min}$ and at $80^{\circ} \mathrm{C}$ for $2 \mathrm{~h}$, shaking at $1000 \mathrm{rpm}$. Samples were ice-cooled for $1 \mathrm{~min}$, purified by centrifugation at $14000 \times \mathrm{g}$ for $15 \mathrm{~min}$ at $4{ }^{\circ} \mathrm{C}$ and the supernatants were recovered and protein quantification was carried out by using the BCA-protein assay kit. Proteins were loaded in $10 \%$ polyacrylamide SDS-PAGE and after electrophoresis, the gel was removed and stained by Coomassie blue according to the manufacturer's instructions (Bio-Rad Laboratories, Hercules, CA, USA) to visualise the distribution of the extracted proteins. The newly described high-temperature extraction conditions in Fig. 1B for FFPE tissues and the previously described conditions at RT conditions in Fig 1A for fresh-frozen tissues have been compared by Coomassie staining. Few differences in band patterns of stained proteins were detected between these methods, suggesting that proteins of different types and quantities were extracted from FFPE tissues sections compared with fresh-frozen tissues. Moreover, the extracted proteins from FFPE are well preserved from low-to-high molecular weights (as seen in the indicated molecular weight marker, MWM). Then, we evaluated our proposed extraction method by mass spectrometry analysis, comparing the sequence coverage achieved on samples obtained with the three extraction conditions described. Several proteins were successfully identified after MALDI-TOF/TOF analysis (4800 Proteomics Analyzer, ABSciex, MA, USA) and database search with MASCOT Version 1.9 from Matrix Science, following the procedure previously described by Calamia et al. 11. As an example, the sequence coverage obtained for human $\alpha$-actin was $39 \%$ in ULB, $40 \%$ in SDS-LB and $43 \%$ in citrate-LB. These results further support that our proposed method for protein extraction from FFPE tissues is also suitable for mass spectrometry analysis. 
A

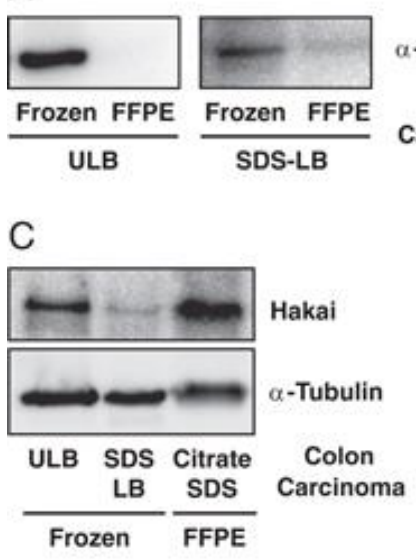

B Mwм

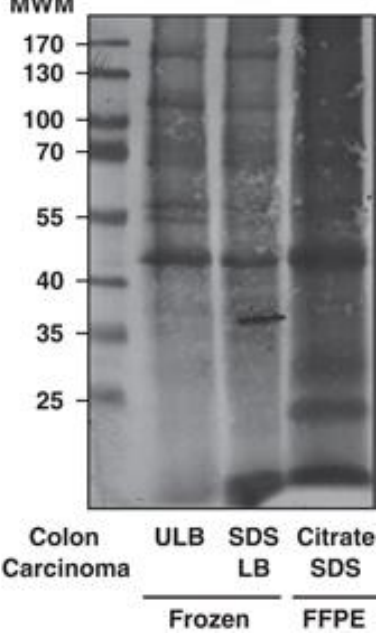

Figure 1. Comparison of the protein extraction protocols from fresh-frozen and FFPE colon carcinoma tissues. (A) Western blotting against $\alpha$-tubulin antibody by extracting protein following the procedure explained at RT by using ULB and SDS-LB buffers. (B) and (C) Coomassie staining and Western blotting against $\alpha$-tubulin and Hakai antibodies by using ULB and SDS-LB at RT and citrate-SDS buffer under the high temperature and conditions proposed. All FFPE samples were used $<1$ year of storage and frozen samples after 5 years of storage. Results are representative of at least three independent experiments.

Next, under the explained extraction conditions used for Fig. 1B, Western blotting was carried out by using $\alpha$-tubulin antibody to confirm the correct normalisation of the samples. High amounts of non-degraded, full-length and equally normalised $\alpha$-tubulin are detected in fresh-frozen and FFPE tissues, as shown in Fig. 1C. In order to evaluate the extraction efficiency from FFPE tissues for other important proteins in colon tumour progression, we analysed Hakai protein expression. Hakai was first identified as a novel E3 ubiquitin ligase that acts on the E-cadherin complex, an epithelial membrane protein that regulates cell-cell contacts. Hakai mediates E-cadherin ubiquitination and degradation altering cell-cell adhesions 12 and plays an important role in proliferation and oncogenesis 13, 14. In Fig. 1C, Hakai was correctly extracted at high temperature from FFPE tissues and similar levels of expression were also detected from fresh-frozen tissues from a different patient in ULB at RT. However, very low detection was seen in the RT extraction with SDS-LB compared with ULB buffer in fresh-frozen tissue from the same patient, further confirming that individual proteins require specific conditions for solubilisation. Moreover, many of the heat-induced antigen-retrieval techniques for intact protein have the disadvantage of reduced or incomplete extraction of membrane proteins. In order to evaluate the extraction performance for this difficult subset of proteins, we analysed E-cadherin expression, which presents high molecular weight $(\sim 120 \mathrm{kDa})$. As expected, E-cadherin was detected by Western blotting in two different FFPE epithelial tissues, colon and kidney (Fig. 2A), further validating this protocol for membrane proteins of high molecular weight, but was not present in epithelial tissues (lymph node and myoma, Fig. 2A, and cerebellum, data not shown). We also analysed Hakai expression in these tissues. Higher amount of Hakai protein was detected in myoma and lymph node (where active proliferative cells are present) compared with normal colon and kidney tissues (Fig. 2A). Furthermore, no detection was found in three human cerebellum blocks analysed (Fig. 2B). In Fig. 2C, an inverse correlation between Hakai and E-cadherin expression was found in two different colon carcinomas (T2 and T3, Fig. 2C), and low expression of both proteins was detected in two colon carcinomas (T1 and T4, Fig. 2C). The meaning of this expression in different colon carcinomas has to be further investigated. Finally, an overview of the workflow of this procedure (Fig. 3A) and the protein yields obtained from the FFPE tissues used is included (Fig. 3B). Taking all these results together, we propose a new method for an efficient extraction of full-length, nondegraded protein from FFPE tissues. This procedure is short time-consuming and cost-effective providing an important tool for the analysis of protein expression through a huge range of clinical samples stored in hospitals worldwide. 

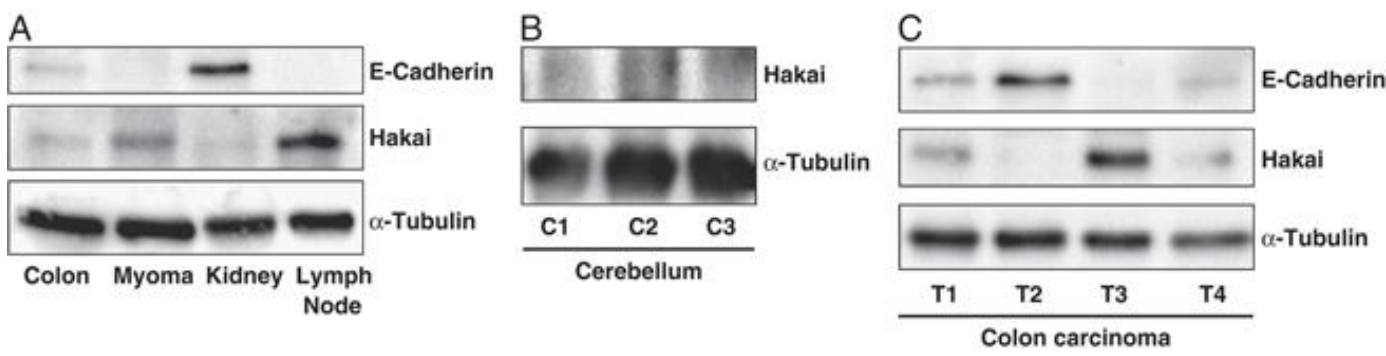

Figure 2. Western blotting against E-cadherin, Hakai and $\alpha$-tubulin from different FFPE tissues. (A) FFPE tissues from colon, myoma, kidney and lymph node. (B) FFPE cerebellum tissues from three different blocks (C1, C2 and C3). (C) FFPE colon carcinoma tissues from different patients (T1, T2, T3 and T4). The procedure was performed by using the explained high-temperature extraction in citrate-SDS buffer. Colon, myoma, lymph node samples in (A), and T3 and T4 colon carcinoma samples in (C) were used after 1 year of storage; the rest of the samples were used after 2 years of storage. Results are representative of at least three independent experiments.

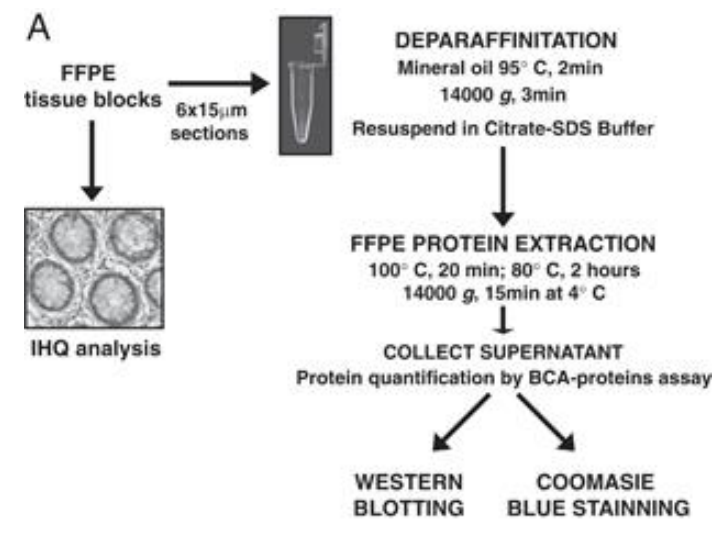

B

\begin{tabular}{|l|c|c|c|}
\hline \multicolumn{1}{|c|}{ Tissue } & $\begin{array}{c}\text { Total Size } \\
\left(\mathrm{mm}^{2}\right)\end{array}$ & $\begin{array}{c}\text { Number } \\
\text { of sections }\end{array}$ & $\begin{array}{c}\text { Protein } \\
\text { Yield }(\mu \mathrm{g})\end{array}$ \\
\hline Normal Colon & $\sim 150$ & 6 & 200 \\
Colon carcinoma & $\sim 200$ & 6 & 250 \\
Myoma & $\sim 150$ & 6 & 180 \\
Kidney & $\sim 250$ & 6 & 300 \\
Lymph Node & $\sim 50$ & 12 & 200 \\
Cerebellum & $\sim 150$ & 6 & 150 \\
\hline
\end{tabular}

Figure 3. Schematic of the workflow (A) and protein yield (B) obtained from protein extraction from FFPE tissues using citrate-SDS buffer.

\section{Acknowledgements}

We thank M. Mayan for critical reading of the manuscript and Y. Fujita for providing Hakai antibody. We also thank Cristina Ruiz-Romero and Valentina Calamia for helpful discussions. T. $\mathrm{R}$. $\mathrm{R}$ is the recipient of a grant from Secretaria Xeral $\mathrm{I}+\mathrm{D}+\mathrm{I}$, Xunta de Galicia, Spain (10CSA916023PR). M. H. C and M. B. are supported by Instituto de Salud Carlos III, Spain (CA09/00116 and CA07/00232). A. F. is supported by Secretaria Xeral I+D+I, Xunta de Galicia, Spain (IPP.08-07). This work is supported by a grant from Conselleria de Sanidade (PS09/24) and from Secretaria Xeral I+D+I (10CSA916023PR) both from Xunta de Galicia, Spain.

The authors have declared no conflict of interest. 


\section{References}

[1] Gilbert, M. T., Haselkom T., Bunce, M., Sanchez, J. J., et al., The isolation of nucleic acids from fixed, paraffin-embedded tissuew-which methods are useful when?. PLoS One 2007, 2, e537.

[2] Ikeda, K., Monden, T., Kanoh, T., Tsujie, M. et al., Extraction and analysis of diagnostically useful proteins from formalin-fixed, paraffin-embedded tissue sections. J. Histochem. Cytochem. 1998, 46, 397-403.

[3] Becker, K. F., Schott, C., Hipp, S., Metzger, V. et al., Quantitative protein analysis from formalin-fixed tissues: implications for translational clinical research and nanoscale molecular diagnosis. J. Pathol. 2007, 211, 370-378.

[4] Nirmalan, N. J., Harnden, P., Selby, P. J., Banks, R. E., Development and validation of a novel protein extraction methodology for quantitation of protein expression in formalin-fixed paraffin-embedded tissues using Western blotting. J. Pathol. 2009, 217, 497-506.

[5] Addis, M. F., Tanca, A., Pagnozzi, D., Crobu, S. et al., Generation of high-quality protein extracts from formalin-fixed, paraffin-embedded tissues. Proteomics 2009, 9 , 3815-3823.

[6] Berg, D., Hipp, S., Malinowsky, K., Böllner, C., Becker, K. F., Molecular profiling of signalling pathways in formalin-fixed and paraffin-embedded cancer tissues. Eur. J. Cancer 2010, 46, 47-55.

[7] Shi, S. R., Liu, C., Balgley, B. M., Lee, C., Taylor, C. R., Protein extraction from formalin-fixed, paraffin-embedded tissue sections: quality evaluation by mass spectrometry. J. Histochem. Cytochem. 2006, 54, 739-743.

[8] Lin, J., Kennedy, S. H., Svarovsky, T., Rogers, J. et al., High-quality genomic DNA extraction from formalin-fixed and paraffin-embedded samples deparaffinized using mineral oil. Anal. Biochem. 2009, 395, 265-267.

[9] Bradford, M. M., A rapid and sensitive method for the quantification of mg quantities of protein. Anal. Chem. 1976, 72, 248-254.

[10] Laemmli, U. K., Cleavage of structural proteins during the assembly of the head of bacteriophage T4. Nature 1970, 227, 680-685.

[11] Calamia, V., Ruiz-Romero, C., Rocha, B., Fernández-Puente, P. et al., Pharmacoproteomic study of the effects of chondroitin and glucosamine sulfate on human articular chondrocytes. Arthritis Res. Ther. 2010, 12, R138.

[12] Fujita, Y., Krause, G., Scheffner, M., Zechner, D. et al., Hakai, a c-Cbl-like protein, ubiquitinates and induces endocytosis of the E-cadherin complex. Nat. Cell Biol. 2002, 4, 222-231.

[13] Figueroa, A., Kotani, H., Toda, Y., Mazan-Mamczarz, K. et al., Novel roles of Hakai in cell proliferation and oncogenesis. Mol. Biol. Cell 2009, 20, 3533-3542.

[14] Figueroa, A., Fujita, Y., Gorospe, M., Hacking RNA: Hakai promotes tumorigenesis by enhancing the RNA-binding function of PSF. Cell Cycle 2009, 8, 3648-3651. 